\title{
Behaviour Analysis in Smart Spaces
}

\author{
Pedro Oliveira \\ Dep. of Informatics and Communications \\ Institute Polytechnic of Bragança \\ Bragança, Portugal \\ Email: poliveira@ipb.pt
}

\author{
Paulo Matos \\ Dep. of Informatics and Communications \\ Institute Polytechnic of Bragança \\ Bragança, Portugal \\ Email:pmatos@ipb.pt
}

\author{
Paulo Novais \\ Department of Informatics \\ University of Minho \\ Braga, Portugal \\ Email:pjon@di.uminho.pt
}

\begin{abstract}
We are on a new era of interaction between persons and physical spaces. Users want that those spaces smartly adapt to their preferences in a transparent way.

This paper describes the process of planning, reasoning and modeling of a Smart Environment with domestic and industrial application, taking advantage of emerging wearable devices on the market (smart watches, fitness trackers, etc.) and newer wireless communication technologies (NFC, BLE, Wi-Fi Direct). Enabling in a noninvasive way for the user, optimize the efficiency, comfort, and safety at the environments.

This approach can be applied in home automation, public spaces and also incorporated at industrial level, to help build smart and autonomous factories.
\end{abstract}

Keywords-smart-spaces; AmI; IoT; wearables;

\section{INTRODUCTION}

As shown in [1] there are new opportunities for research in the field of smart environments that should be explored. In particular the concepts of smart homes and home automation [2], currently in growing expansion in the scientific and research point of view, as the market demands for better solutions in this field. The aim is to take advantage of emerging technologies available in the market that support the so-called wearable devices [3] [4], and the non-invasive particularity of these to, in an autonomous way, adapt the environment to the comfort parameters of each user (e.g. thermal, acoustic, air quality, light, sun exposure). Provide comfort according to the preferences of each individual, is a challenge and an opportunity to create innovative solutions and new paradigms in the context of Intelligent Environments [5] [6].

Behaviour analysis in smart environments is performed mainly using data collected from the sensors dispersed throughout the environment [7] [8]. As concluded in [9], the perfect learning system for smart environments has not yet been found, and each new valuable contribution in this field puts us one step closer to the true concept of intelligent environments [10]. It is also referred the need and the challenge of establishing a new effective paradigm for ambient intelligence, where the focus becomes the user and the ability to manage the complexity and richness of everyday human life [6] [9]. A recurring challenge in this field is the management of conflicts of interest among the several users of the space [11] [12], that in this work will be addressed through the use of multi-agent systems, as well as the real-time data acquisition of the user information (e.g. body temperature, pulse) [13] [14]. It will be possible, making use of technologies and emerging wearable devices available on the market (e.g. smartwatches, smartphones, fitness trackers) [11], to focus the data collection process on the user, always taking into account that it will be a non-invasive process. This will significantly leverage/enrich the decision-making process and overtake the physical limits so far imposed by the need of sensors statically placed in a space [15] [16]. After analyzing the state of the art, we can point out the scientific innovation and contribution that this project can bring to this area: conceive and design new solutions and paradigms that contribute to turn into a reality the concept of intelligent environments. Besides the scientific contributions, this work will produce relevant results with industrial application since it will be carried out jointly with an industrial partner. Prior to this proposal, this partner conducted several market and search studies. The conclusion was that the national market does not offer solutions with such level of intelligence, user-oriented or based on non-intrusive techniques of data acquisition. At an international level there are products with some features implemented, but which always need the programming and configuration of the product by the user, are oriented to the spaces and not to the individual or do not have sufficient artificial intelligence that enables predictive capabilities and improvements in the effectiveness of the product in decisionmaking process.

This paper is structured in the following way. Section II starts with the description of the challenge to be addressed, the data managed by this system, and ends with the detailed description of the goals that the system have to achieve. Next, in section III is proposed and detailed the general requirements of a possible solution and a system architecture. Section IV ends with conclusions and possible future work.

\section{MATERiAls AND Methods}

This section describes the problem statement, and a detailed description of the challenge. 


\section{A. Problem Statement}

The problem/challenge, need or, on the economic perspective, opportunity, which aims to overcome, can be defined in general terms as the difficulty in optimizing automatic, transparent and in a non-invasive way for the user, the comfort conditions, both at domestic and professional level or even in the use of public spaces. When it comes to comfort, we can also talk about security, productivity or other.

This challenge relatively to users, currently has as main difficulty, the mobility of people, the disparity of habits, schedules and every individual comfort preferences. The same is aggravated when depending on physiological conditions, derived from a large number of factors (tiredness, mood, etc.), user preferences often suffer significant changes, that current systems can not measure.

Contextualize user preferences, it is a process involving many variables and different dimensions, which makes this a problem of high complexity.

In addition to the physiological conditions mentioned above, there are two critical/essential dimensions, these are the space (user location) and time. In the case of the space can be as an example, the differences between the preferences of a personal, professional, recreational or other environment. Contextualize the user location is essential to optimize the conditions of comfort and contribute to the performance and effectiveness of the solution.

The time dimension is equally critical. Because the comfort preferences will change over the course of the day, as well as the week or even the year. For example, the comfort preferences may be different between daytime and nighttime, or between weekday and weekend/holidays. In this dimension, it is also important to assess the changes over the year, that will have the influence of the seasons, which naturally also change quite the user's comfort preferences.

In this paradigm, which is intended to be the superlative of the comfort, there are at least these three dimensions: time, space and user comfort preferences.

The time and space dimensions are critical to contextualize the user's personal preferences, and provide the necessary information that will allow assess future preferences in a useful time.

It should be noted that systems that promote comfort (air conditioning, heating, air oxygenation, etc.) have latencies (inertia) of operation. Early prediction of user presence and their comfort preferences allows to surpass this latency. Thus starting its functioning in due time, to be achieved the comfort conditions. The same solution also allows to optimize energy savings associated with such equipment, since it allows to predict when the systems may be disabled or placed to operate more efficiently. This savings can be defined in various dimensions such as energy, maintenance, useful life period, etc.
And these were the main issues of the problem, of course there are accessory issues, such as the management of more than one user in the same space, among others that naturally may arise and should be addressed.

Figure 1, shows the scenario of an environment where it intends to develop this work. Explaining this figure, it can be seen the user who through its different devices (smartphone, wearable, and other compatible) communicates with the system, and for that can be used different technologies (Wi-Fi Direct, Near Field Control, Bluetooth Low Energy). Next, the system performs communication with the Cloud, to validate the information. And then the system will perform the management of the different components in the environment (climatization systems, security systems, other smart systems).

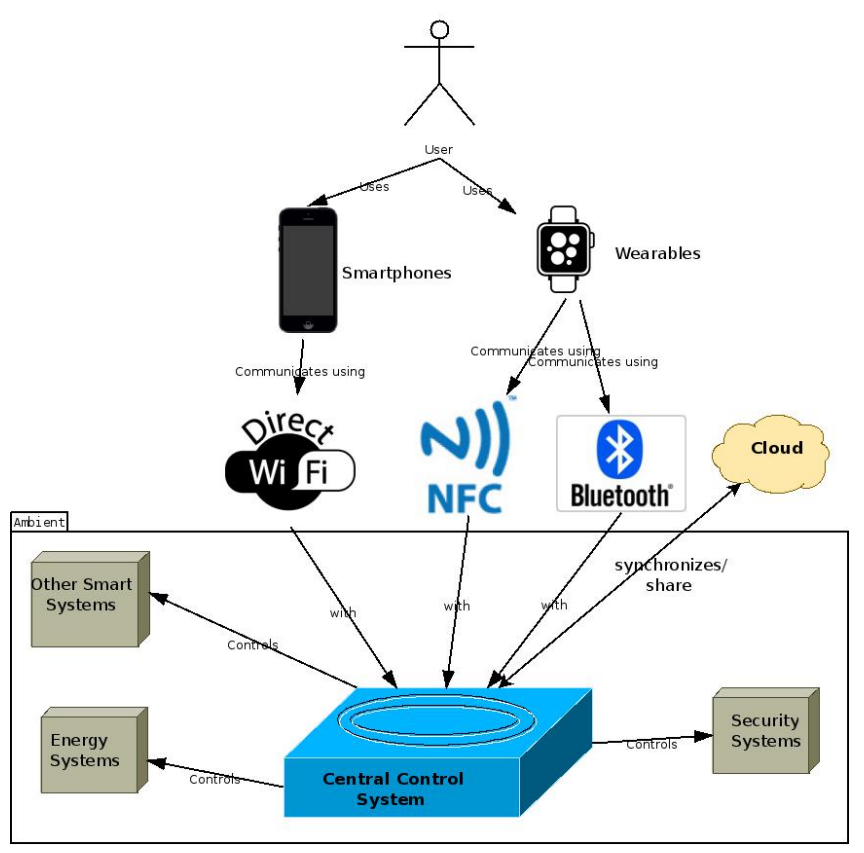

Figure 1. Problem Statement

This scenario, represents an environment at a given location and at a given point in time. As mentioned above, for a proper solution that fits all users, it is necessary to consider the user mobility. This implies taking into account the time and space dimensions.

As such, for a better understanding of the overall scenario in the user's daily life, figure 2 contextualizes the temporal and space dimensions present in this problem and already mentioned above. We can see that different user locations, combined with time context, naturally results in an environment with different characteristics. This kind of global scenario should also be addressed in this project.

\section{B. Overall Description}

This section deals with the goals to be achieved, and detail the plan defined to solve the challenge. 


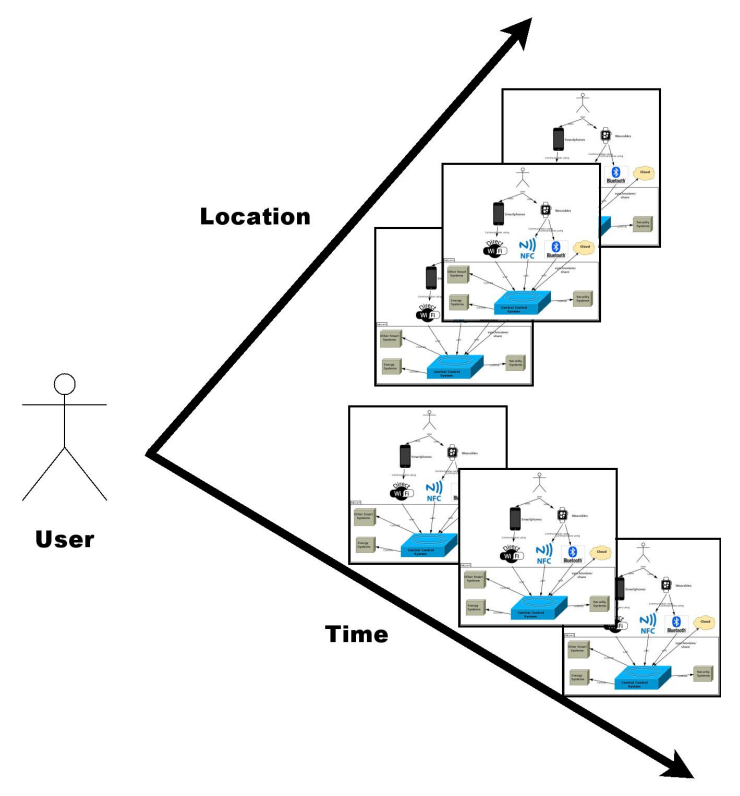

Figure 2. Contextualization of Time/Environment Dimensions

1) Goals: The aim of this project is to create a solution that takes advantage of emerging technologies on the market that support wearable devices (e.g. smartwatches, smartphones, fitness trackers) and the non-invasive characteristic of these, for collecting data in an autonomous and transparent way and without any need of intervention by the user. And with that information assist the decision-making process of comfort systems to adapt the environment to suit the comfort preferences of each user (e.g. thermal, acoustic, air quality, lighting, sun exposure).

The main goal that this project aims to achieve is to set a base architecture for a non-invasive system that takes advantage of emerging technologies and wearable devices data collection (smartwatches, smartphones, fitness trackers) for the intended purpose. And using intelligent agents [17], to represent the different stakeholders, contexts and dimensions of the problem, who cooperate to achieve the optimal solution.

2) Detailed Description: This subsection refers several times, the concept of space and environment. As such, it is necessary to contextualize them, prior to use.

By space means the global context where the user is located, namely the building, housing or public space.

On the other hand, environment refers to the specific location within a given space, namely the internal division, as the hall, room, or office.

Being the space naturally wider, and the environment being inserted in the space.

The process and learning model proposed to the system, is intended to be scientifically innovative, taking advantage of the latest research in this field and combining multiple factors and technologies described below:
- Use dynamically scaled priorities rules, which must have the information, considered essential for the correct functioning of the system, including the limits for the different parameters, like system reliability or user safety.

- Use of multi-agent systems representing the different entities involved in the negotiation process, allowing an efficient outcome in different situations.

- Use of sensors collected information, and using machine learning techniques, including Sequence Discovery, Fuzzy Logic, Genetic Programming, Multi-Layer Perceptron, as described in [18], get information about user's habits in the environment.

- Context awareness, as described above since the context is entirely relevant in such systems. In the literature, context sensitive systems are described as members of the ubiquitous computing environments. These systems take into account the different information related to location, environment, resources, users and the relationship between each of them. They intend to get personalized decisions depending on contextual factors. So it can happen different decisions, to the same situation in different contexts [19]. The contextualization elements, can be obtained in several ways as described in [20].

- Use of logical sensors, there are three types of sensors used to assess the context in such systems: physical, virtual and logical. Physical sensors capture the context of the information, and are dispersed throughout the environment. Virtual sensors are specified and configured, to collect contextual information using as sources applications or services. Logical sensors, through the combination of physical and virtual sensors, intended to determine logical values for the attributes to be collected. For a smart environment contextualization, the attributes are typically collected using the three types of sensors depending on the contextual nature within the smart environments.

That said, the practical applicability of this project, should result in the complete specification of an intelligent environment. Namely to ensure a solution that covers the following features:

- User identification;

- Detection and characterization of the user at the space;

- Detection and characterization of the user in the environment;

- Preferences conflict management in the environment;

These points are seen in detail in the following subsections.

3) User identification: In this project, the user identification, is one of the essential tasks and it should be analyzed carefully later. However in a first approach, it is planned that there are two situations, explained below: 
- User ID sharing: in this situation, when the user enters in the environment, the devices that are with him (smatphone, wearables, etc) pass the user ID to the system that controls the environment. The system, validates the ID in the Cloud, and from this gets the user's preference card. The system will then use the card information received, to adapt the environmental comfort conditions, using the automation available in the environment.

In this case the system is permanently connected to the Internet, so that is allowed access to the Cloud.

- User preferences card sharing: In this case the user when enter the environment, share directly with the system, its preferences card, with the card available in the compatible device (smartphone, wearable, etc.).

The system collects data from these preferences and adapts, as in the previous case, the environment comfort conditions, using for that purpose the automated systems available in the environment.

In this case, the system does not require an Internet connection, all the process may be performed offline.

Both situations assume that the user has no part in the process, which is completely transparent to him.

The use case diagram present in figure 3 , illustrate the operating modes provided for the implementation of the user detection process, and sharing of his preferences card with the system on the environment.

Initially are provided two cases of use, depending on the network connection status of the system. For systems located in environments where there is no connection to the Cloud, or where the system is offline, the user device share directly with the system, its preferences card, continually the system proceeds to the environment adjustment according to existing needs and different valences on the environment. In case of missing valences, the respective shared preference will be discarded.

The other case implies a permanent connection of the system to the Cloud. In this situation, the user does not directly share his preferences card, but his user ID, and this ID is validated by the system in the Cloud. Upon successful validation carried out by the system, it will be returned from the Cloud the user preferences card. After the correct reception of this by the system, it will affect the management of the environment in the same way mentioned above.

In figure 4, an example of an environment is illustrated to demonstrate the use cases described above. Note that the communication processes represented in this figure on arrow format is expected to be transparent to the user and completely independent of its intervention.

After the validation in the Cloud of the user ID, the respective preferences card is downloaded into the system, and the control is made automatically by the system, adjusting all the preferences existing in the environment. In this example, will be adjusted lighting, radiator and air conditioning.

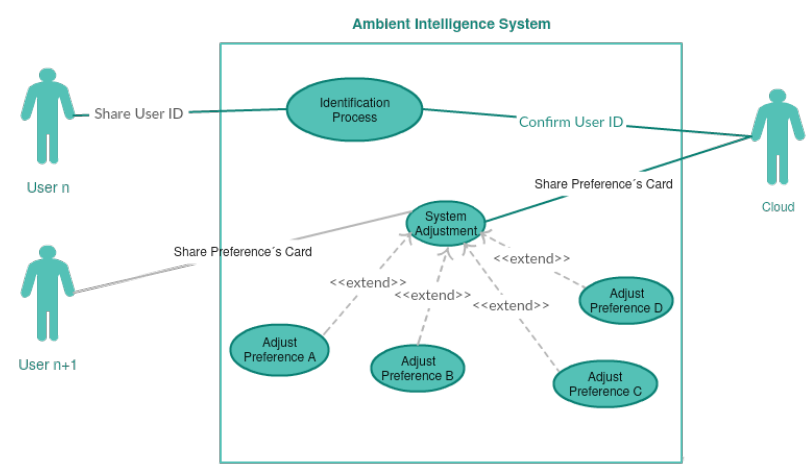

Figure 3. AmI System - Use Case diagram

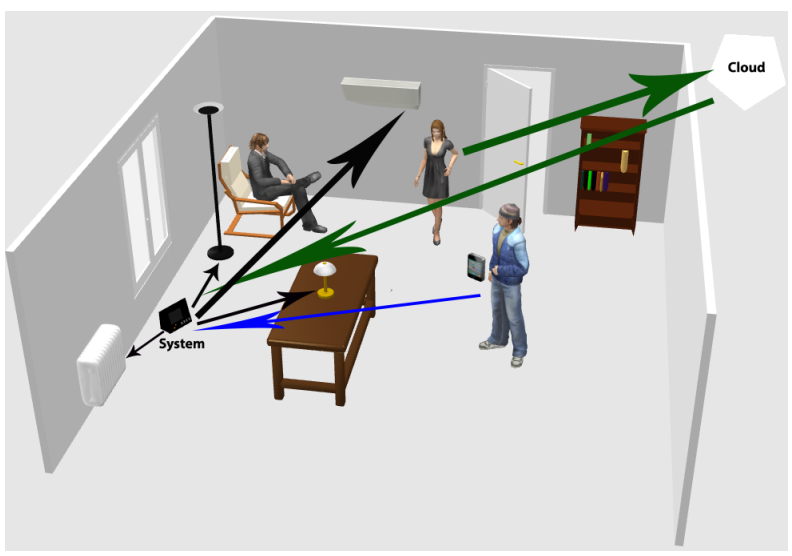

Figure 4. AmI System - Communication process

4) Detection and characterization of the user at the space: In their daily lives, the user moves through different spaces and situations where although there are routines, it becomes difficult to identify accurately and efficiently which are their movements and location. In this sense, having the real-time user's location within a particular space, is an extreme valuable information regarding the environment optimization using forecast methods.

Previously, this information was just captured through the use of presence sensors disposed statically, and had several gaps, including the existence of many points where they did not obtain information (blind spots). This type of sensor requires constant maintenance, and its prior application in areas where it was intended to have this kind of information, that with the current mobility of people, often prevented the gathering information on new areas of use, due to impossibility of prior knowledge of user presence in them [9].

At figure 5, is illustrated an example of context as described above. This figure shows a space divided in different environments. As can be seen, naturally users move between environments, and so there may be environments with one or more users, as well empty environments. 
Collect and evaluate this type of information is relevant, to optimize the conditions of space in general and of the environment in particular.

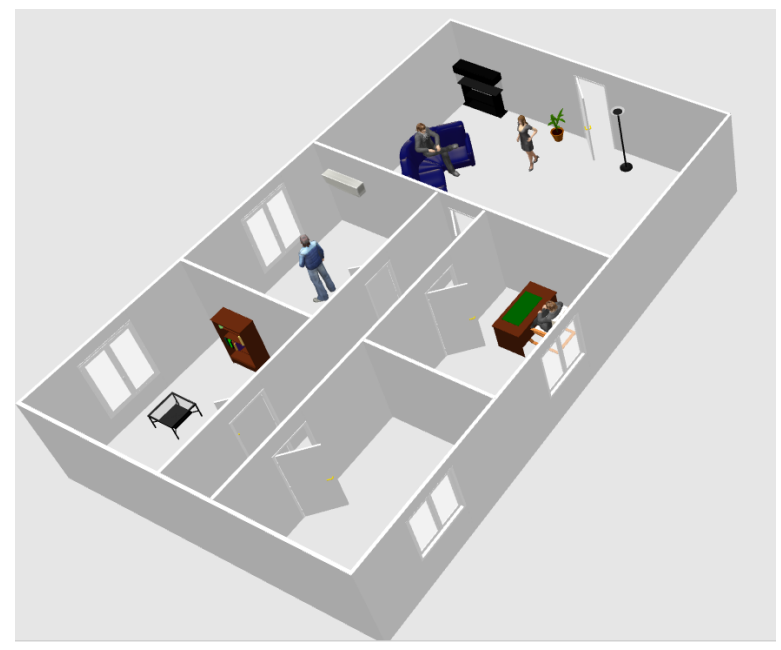

Figure 5. Detection and characterization of the user at the space

5) Detection and characterization of the user in the environment: In addition to the user's location in space, described above. It may also be hugely important to get, the specific location of the user within the environment. Because this distance will have influence, especially in the specification of their conditions of comfort, managing to have the knowledge of the distance from the sources of heating/cooling, as well as, other equipment's available in the environment that interfere with the comfort conditions.

With this information, response and configuration of these devices can be adjusted in real time to increase the effectiveness of their performances, both in terms of service quality, such as energy efficiency, which will result in an obvious and desired reduction of energy costs for the final consumer.

This type of proximity information indoors, was previously technologically unfeasible, especially due to the costs of implementing a solution like that.

Currently the type of existing sensors and technologies, particularly in terms of Bluetooth Low Energy (BLE) [21], are fully implemented in the market and at reduced cost to the user, which enables the optimization in a relevant way, and to overcome the type of problems identified above.

In figure 6, is illustrated an example of context as described above. This describes an example of an environment, where there are two users. The proposed system should identify them and measure the distance of these to the systems involved in comfort conditions.

6) Preferences conflict management in the environment: Another problem that stays current in research, is the management of conflict preferences, which also applies in the AmI area.

It is known that each person is unique in its individuality,

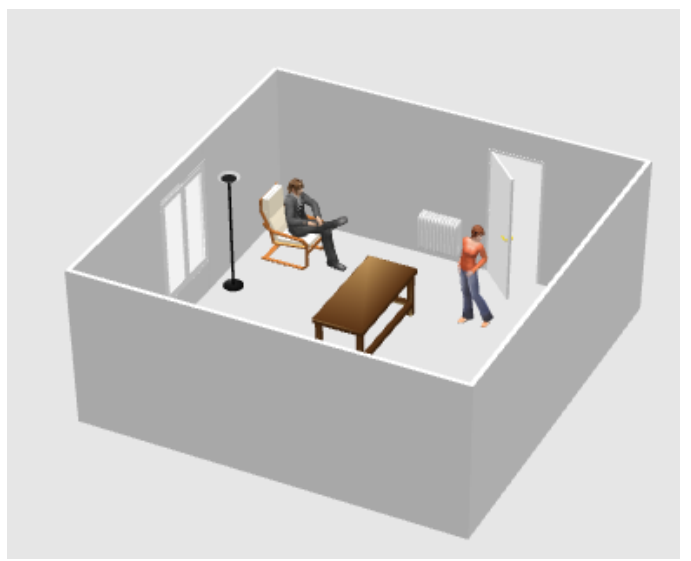

Figure 6. Detection and characterization of the user in the environment

and this naturally translates in his comfort preferences, that also vary with the physiological and physical conditions of each one. Namely there may be incompatibilities between the natural comfort preferences of different users that are in the same environment.

To overcome the incompatibility, it must exist obvious compromises between the preferences of the different stakeholders.

In this field of conflict management, there are already quite work, currently undergoing. Most of it through the use of agents that carry out negotiations for the resolution of potential conflicts [22].

In figure 7, is illustrated an example of context as described above. Can be verified an environment where there are three users, which will invariably have different preferences. What will lead the system to act in order to overcome the best of these differences.

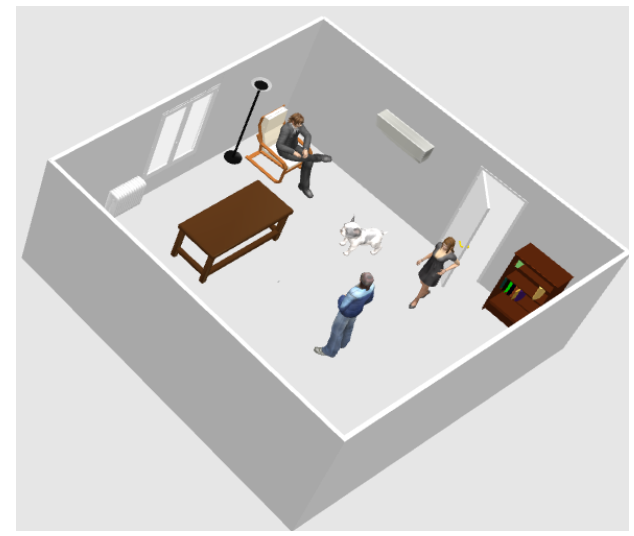

Figure 7. Preferences conflict management in the environment

\section{RESULTS}

This section start with the general requirements and relevant information that needs to be addressed by the proposed solution, and finishes with a proposed system architecture. 


\section{A. General Requirements}

1) Required and Relevant Data: Any intelligent environment situation, necessarily implies a process of collection/acquisition of data. It will be using this information, that the environment/space will optimize their decisions in a autonomously and intelligently way.

The existing literature already points out several ways of obtaining data, in this kind of spaces. Imperatively the most traditional way on the market are the sensors, these allow to collect different types of information (temperature, humidity, etc.) [9]. These sensors can be isolated, dispersed throughout the environment or integrated into other devices, that users use and carry with them in their daily routine, in a noninvasive way. These sensors are currently in devices such as smart watches, fitness bracelets, smartphones, etc. This ubiquity of IoT will enable a constant reading of the information needed for use by these intelligent environments. Especially with the miniaturization of this type of sensors, and the technology evolution that allows communication between them and the Internet, and taking advantage of a low power that they require.

Table I

WEARABLES - COMMON SENSOR'S INFORMATION [23]

\begin{tabular}{|c|c|}
\hline Sensor & Information \\
\hline Pedometer & Steps counting \\
\hline EMG & Muscle activity walking \\
\hline EEG & Brain activity signals \\
\hline ECG & ECG signals \\
\hline PPG & Volumetric measurement of blood \\
\hline SpO2 & Oxygen saturation (SpO2) \\
\hline Bio-sensor needle & Blood Glucose level \\
\hline Heart Rate & Number of contractions of the heart \\
\hline Temperature & Body Temperature \\
\hline Sweat & Water evaporation rate from the skin \\
\hline
\end{tabular}

There is also the fact, that are now being studied and are technologically, and economically viable, sensors for physiological analysis (transpiration, body temperature, glucose, heart rate, etc.) as indicated in table I that could be most valuable for a prediction and medical examination of each individual.

This control, was previously only possible in appropriate environments, and using expensive and large size devices, which is not allowed in any way, that each person could perform their own control independently and regardless of their technical knowledge.

The acquired physiological information of wearable devices, is of great relevance. Currently wearables market, collect certain indicators, as previously indicated in table I, that using health models, already well validated scientifically, make possible to determine the comfort satisfaction of the user. This information is relevant, to change the comfort conditions of a particular environment. It has only recently become possible and practicable, since only now technology has enabled miniaturization of this type of sensors, and so these are integrated into wearable devices with a commercial value that is accessible to the most users.

In addition to these factors, using other fields of study, and in particular concerning indoor location, can be used the dispersion of terrestrial magnetic field, to have very high accuracies in indoors location [24] [25]. This is as important, as it is to get the distance between the user and the elements that allow the comfort conditions (heating, cooling, air quality). It allows to optimize the operation of these elements. This information is even more important on large size environments, containing a large number of comfort elements, where they may be adjusted taking into account the dispersion of the users in the environment.

2) Preference's Card: Each person has their comfort preferences and, of course, these vary with a number of physiological and other factors intrinsic to each user, as well as the time of day, mood, etc.

Also each person as an individual, has different comfort preferences in different situations. This is so, a number of unpredictable situations.

This requires that each person, whenever interacts with a new environment, proceed with a manual configuration of him, which is the current form that the user has to adjust the environment to their needs.

This being the era of automation in the most diverse tasks, also in this field it has created the need for the existence of automation, because this type of situation happens in the daily life of any person. And this improvement, in addition to an increase of comfort, bring a number of added value, such as time savings of the people, greater economic savings as a result of increased energy efficiency, as well as saving in the equipment maintenance.

Operationalize this situation requires the definition of a set of parameters, which are deemed essential to set personal comfort. This set will be called "preferences card" of the user. This will be populated with the relevant information, which is expected to be also dynamically updated by the system without user intervention.

This is possible using learning mechanisms, that over time will learn the user's preferences, becoming these values stable after the learning process reaches the maturity point.

This card includes a comprehensive number of preferences, which are obviously not possible to control in all environments, because the lack of compatible equipment's on the environment, or even because the configuration in the environment is not applicable. In this case, the preferences not supported by the system are discarded.

As shown in figure 1, several technologies, support this implementation and allow the communication process between user devices and the system on the environment. These technologies are in a relatively stable state, but still in an evolutionary process to obtain better performances at 
various levels (data transmission rates, range, consumption).

Will be studied the feasibility of using the technology Near Field Communication (NFC) [26], Bluetooth Low Energy (BLE) [21] and Wi-Fi Direct [27]. Not being put aside the use of several technologies simultaneously, if they complement each other in different situations.

The preferences card will initially have the characteristics defined in table II, since such are currently the most common in the user's daily life. It is however a scalable card, according to the needs require. The default values for each preference, are defined at the first time by the user, using a smartphone app.The evolution and adaptation of the preference card, can be done at each time by the user using the smartphone app. Also in a automatic way the values are also updated using the feedback retrieved by each local system where the user interacts.

Table II

PREFERENCE'S CARD

\begin{tabular}{|c|c|c|}
\hline Preference & Example & Unities \\
\hline Temperature & 20 & $\mathrm{C} / \mathrm{F}$ \\
\hline Iluminance & 40 & Lux (lx) \\
\hline Brightness & 100 & Watts/cm2 \\
\hline Relative Humidity & 45 & $\%$ \\
\hline Musical Genre & Pop & String \\
\hline Musical Playlist & (Adele, U2) & String \\
\hline
\end{tabular}

3) System Information: On the other hand, as mentioned in section II-A, the system will also have to provide a range of information, including on security issues, which enable alert wherever the reference values taken as safe are exceeded. In the examples shown on table III are represented the reference values for the most common gases that would jeopardize the safety of users inside closed environments.

Also, to have the context of temporal and location dimension represented in figure 2, it is also stored the date, time, location, and the local GPS coordinates. All this information, obviously refers to the location where the system is installed. Table III exemplifies the information that will be available in the system. This information is scalable and adaptable to the concrete requirements.

Table III SYSTEM INFORMATION

\begin{tabular}{|c|c|c|}
\hline Characteristic & Example & Unities \\
\hline Time & $20: 00$ & Hours \\
\hline Date & $20 / 11 / 2015$ & Date \\
\hline Local & Work & String \\
\hline GPS Coordinates & $39,399872,-8,224454$ & String \\
\hline Carbon dioxide $(\mathrm{CO} 2)$ & 500 & ppmv \\
\hline Carbon monoxide $(\mathrm{CO})$ & 2 & ppmv \\
\hline
\end{tabular}

\section{B. System Architecture}

At figure 8 is presented the system architecture proposed to solve the challenge described above. With these architecture it is possible to achieve all the use cases demonstrated in the respective diagram showed at figure 3.

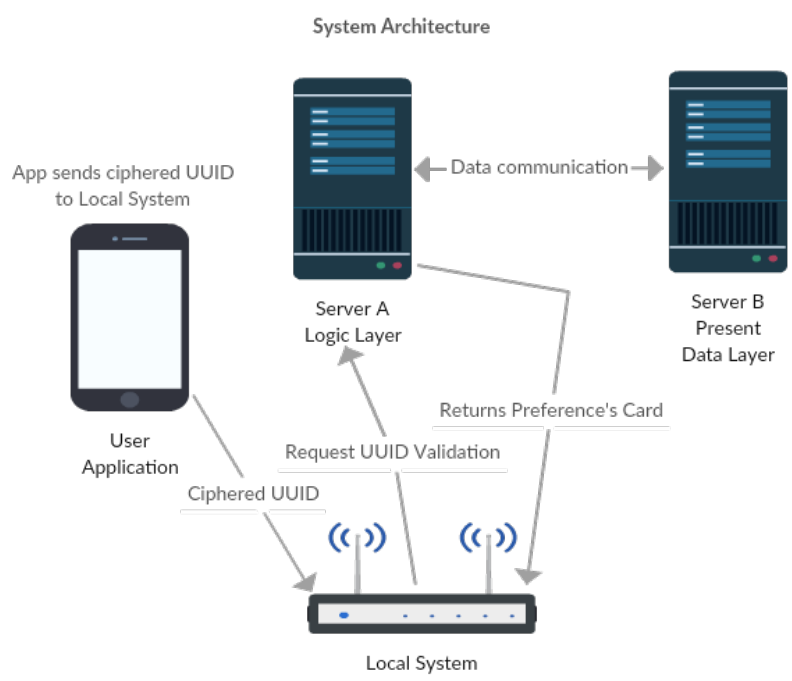

Figure 8. System Architecture

\section{Discussion AND CONCLUSIONS}

This project combines the use of the latest wireless communication technology (NFC, BLE, Wi-Fi Direct) with emerging wearable devices, and therefore optimize the everyday people lives and the industrial production environments.

Is defined as a truly innovative project and fully applicable to industrial and domestic level. And with really high market value, such as envisage diverse reports of global reference consultants (Gartner [28], McKinsey [29], Business Insider [30]) in its most recent reports which include projections for the (2015-2020) period.

For future work, we have identified the need to develop solutions that enable communication between the application and the local system, using different communication technologies without user interaction.

Currently some mobile platforms do not provide direct access to SDK's that enable the development of applications for use this type of communication technologies. Some extra work must be done to overcome this constraint, and get a transparent use solution for every user.

\section{REFERENCES}

[1] E. H. Aarts and B. E. de Ruyter, "New research perspectives on ambient intelligence." JAISE, vol. 1, no. 1, pp. 5-14, 2009.

[2] E. Aarts and F. Grotenhuis, "Ambient intelligence 2.0: Towards synergetic prosperity," Journal of Ambient Intelligence and Smart Environments, vol. 3, no. 1, pp. 3-11, 2011. 
[3] E. Fleisch, "What is the internet of things? an economic perspective," Economics, Management, and Financial Markets, no. 2, pp. 125-157, 2010.

[4] K. Gama, L. Touseau, and D. Donsez, "Combining heterogeneous service technologies for building an internet of things middleware," Computer Communications, vol. 35, no. 4, pp. 405-417, 2012.

[5] J. C. Augusto, V. Callaghan, D. Cook, A. Kameas, and I. Satoh, "Intelligent environments: a manifesto," HumanCentric Computing and Information Sciences, vol. 3, no. 1, pp. 1-18, 2013.

[6] D. Preuveneers and P. Novais, "A survey of software engineering best practices for the development of smart applications in ambient intelligence," Journal of Ambient Intelligence and Smart Environments, vol. 4, no. 3, pp. 149-162, 2012.

[7] A. Aztiria, "Learning frequent behaviours of the users in intelligent environments," Journal of Ambient Intelligence and Smart Environments, vol. 2, no. 4, pp. 435-436, 2010.

[8] A. Aztiria, J. C. Augusto, R. Basagoiti, A. Izaguirre, and D. J. Cook, "Discovering frequent user-environment interactions in intelligent environments," Personal and Ubiquitous Computing, vol. 16, no. 1, pp. 91-103, 2012.

[9] A. Aztiria, A. Izaguirre, and J. C. Augusto, "Learning patterns in ambient intelligence environments: a survey," Artificial Intelligence Review, vol. 34, no. 1, pp. 35-51, 2010.

[10] D. J. Cook, "Learning setting-generalized activity models for smart spaces," IEEE intelligent systems, vol. 2010, no. 99, p. 1,2010 .

[11] M. Gomes, T. Oliveira, D. Carneiro, P. Novais, and J. Neves, "Studying the effects of stress on negotiation behavior," Cybernetics and Systems, vol. 45, no. 3, pp. 279-291, 2014.

[12] F. Rivera-Illingworth, V. Callaghan, and H. Hagras, "Detection of normal and novel behaviours in ubiquitous domestic environments," The Computer Journal, vol. 53, no. 2, pp. 142-151, 2010.

[13] D. Carneiro, M. Gomes, Â. Costa, P. Novais, and J. Neves, "Enriching conflict resolution environments with the provision of context information," Expert Systems, 2013.

[14] S. Iyengar, R. R. Brooks, and G. Karjoth, "International journal of distributed sensor networks," International Journal of Distributed Sensor Networks, vol. 4, no. 1, pp. 1-3, 2008.

[15] M. Chui, M. Löffler, and R. Roberts, "The internet of things," McKinsey Quarterly, vol. 2, no. 2010, pp. 1-9, 2010.

[16] D. Bandyopadhyay and J. Sen, "Internet of things: Applications and challenges in technology and standardization," Wireless Personal Communications, vol. 58, no. 1, pp. 4969, 2011.

[17] F. Bellifemine, A. Poggi, and G. Rimassa, "Developing multiagent systems with jade," in Intelligent Agents VII Agent Theories Architectures and Languages. Springer, 2001, pp. 89-103.
[18] K. I.-K. Wang, W. H. Abdulla, and Z. Salcic, "Ambient intelligence platform using multi-agent system and mobile ubiquitous hardware," Pervasive and Mobile Computing, vol. 5, no. 5, pp. 558-573, 2009.

[19] B. Schilit, N. Adams, and R. Want, "Context-aware computing applications," in Mobile Computing Systems and Applications, 1994. WMCSA 1994. First Workshop on. IEEE, 1994, pp. 85-90.

[20] M. Baldauf, S. Dustdar, and F. Rosenberg, "A survey on context-aware systems," International Journal of Ad Hoc and Ubiquitous Computing, vol. 2, no. 4, pp. 263-277, 2007.

[21] S. Bluetooth, "Bluetooth core specification version 4.0," Specification of the Bluetooth System, 2010.

[22] S. D. McArthur, E. M. Davidson, V. M. Catterson, A. L. Dimeas, N. D. Hatziargyriou, F. Ponci, and T. Funabashi, "Multi-agent systems for power engineering applicationspart ii: Technologies, standards, and tools for building multi-agent systems," Power Systems, IEEE Transactions on, vol. 22, no. 4 , pp. 1753-1759, 2007.

[23] A. Rehman, M. Mustafa, I. Israr, and M. Yaqoob, "Survey of wearable sensors with comparative study of noise reduction ecg filters," Int. J. Com. Net. Tech, vol. 1, no. 1, pp. 61-81, 2013.

[24] J. A. Tauber et al., "Indoor location systems for pervasive computing," Massachusetts Institute of Technology. Area exam report, 2002.

[25] W. Storms, J. Shockley, and J. Raquet, "Magnetic field navigation in an indoor environment," in Ubiquitous Positioning Indoor Navigation and Location Based Service (UPINLBS), 2010. IEEE, 2010, pp. 1-10.

[26] R. Want, "Near field communication," IEEE Pervasive Computing, no. 3, pp. 4-7, 2011.

[27] D. Camps-Mur, A. Garcia-Saavedra, and P. Serrano, "Deviceto-device communications with wi-fi direct: overview and experimentation," Wireless Communications, IEEE, vol. 20, no. 3, pp. 96-104, 2013.

[28] V. Alfonso, G. Eric, C. Sree, and F. Jouni, "Market trends: Tsps must invest in the rapidly evolving iot ecosystems now," 2013.

[29] J. Manyika, M. Chui, P. Bisson, J. Woetzel, R. Dobbs, J. Bughin, and D. Aharon, "The internet of things: Mapping the value beyond the hype," 2015.

[30] B. Insider, "The internet of things 2015 report: Examining how the iot will affect the world," 2015. [Online]. Available: http://www.businessinsider.com/internet-of-things2015-forecasts-of-the-industrial-iot-connected-home-andmore-2015-10 